\title{
All-Optical Diode Action in Quasiperiodic Fibonacci Nanostructures
}

\author{
A. NAMDAR ${ }^{a, *}$ AND F. EBADI-GARJAN ${ }^{b}$ \\ ${ }^{a}$ Faculty of Physics, University of Tabriz, Tabriz, Iran \\ ${ }^{b}$ Physics Department, Azarbaijan University of Tarbiat Moallem, Tabriz, Iran
}

(Received June 7, 2012)

\begin{abstract}
We theoretically investigate the feasibility of nonreciprocal light propagation by using the unique features of quasiperiodic one-dimensional photonic crystals. The intrinsic spatial asymmetry of the Fibonacci sequence leads to strong non-reciprocal electric field distribution, so the nonlinear transmission becomes sensitive to the propagation direction. In this work we emphasise on bistability and all optical switching where the switching threshold for one propagation direction is lower than that for the other. In addition we demonstrate that this structure exhibits high transmission due to the existence of high transmission resonances in its linear transmission spectrum, and these features allow creating an all-optical diode.
\end{abstract}

DOI: $10.12693 /$ APhysPolA.123.45

PACS: 42.65.-k, 42.70.-a, 78.20.-e

\section{Introduction}

An all-optical diode (AOD) is a spatially nonreciprocal device that in the ideal case, for specific wavelengths and power densities allows the total transmission of light along the forward direction and totally inhibits light propagation along the backward direction. AODs have attracted a lot of attention due to their key role in designing the key components for the next generation of all-optical signal processing. AODs are quite comparable with electronic diodes which are widely used in computers for the processing of electric signals. Replacing relatively slow electrons with photons as carriers of information would considerably increase the speed and the bandwidth of telecommunication systems $[1,2]$, thus constructing highly efficient AODs leads to a real revolution of the telecommunication industry.

To this end, a variety of structures have been used, including photonic crystal (PC) waveguides with embedded nonlinear defects [3], asymmetric multilayer structure consisting of two materials with positive and negative refractive indices [4], structures with an axially asymmetric nonlinear absorption [5], the Thue-Morse superlattices [1], heterostructures consisting of metal layers [2], nanocomposite photonic crystal microcavities [6].

The operation of nonlinear photonic multilayer based optical diode relies on two principles. First, the structure must be spatially asymmetric so that the resonant mode couples more strongly to the wave impinging from one side than from the other side. Second, the structure should be strongly nonlinear. Regardless of the strong asymmetry of the considered lattice optical diode action is impossible in the linear regime. A spectral resonance is usually employed to enhance the nonlinear interaction, also providing the structure with a highly transmissive state which is very sensitive to parameter variations. This causes a direction dependent refractive index change due to the Kerr nonlinearity [7].

*corresponding author; e-mail: a.namdar@tabrizu.ac.ir
To increase the structure's sensitivity to the direction of incidence, it is necessary to increase the spatial asymmetry of the structure. The concept of quasiperiodicity was transferred to photonic crystals and proved to be of great value for many practical purposes [8]. Photonic quasicrystals are deterministically generated dielectric structures with a non-periodic modulation of the refractive index. In the one-dimensional case, they can be formed by stacking together dielectric layers of several different types according to the substitutional sequence under investigation (Rudin-Shapiro, Thue-Morse, Fibonacci, etc.) [9].

The Fibonacci sequence is of particular importance, since it leads to the existence of two incommensurable periods in the spatial spectrum of the structure. Such behavior is typical of sequence with a so-called pure point spectrum, which makes the Fibonacci sequence truly quasiperiodic, as a consequence of the appearance of Bragg-like peaks in the spatial spectrum [10]. In this paper we study the transmission properties of the Fibonacci quasiperiodic nanostructures composed of linear and nonlinear layers.

The plan of this work is as follows: in Sect. 2 we study the linear transmission behavior for different generation numbers of the Fibonacci quasiperiodic nanostructures. Then in Sect. 3 we investigate the possibility of optical diode action for considered structures. Finally, a brief summary is given in Sect. 4 .

\section{Linear transmission of Fibonacci photonic crystals}

There are two main approaches to generate 1D quasi-periodic sequence. The first makes use of a projection from a higher-dimensional space, while the second employs the so-called substitutional sequences [11]. Being strictly quasi-periodic, the Fibonacci sequence can be obtained by using both of the above methods. However, these two approaches are not equivalent to each other, and the use of substitutional sequences tends to be a more general procedure [12], so it can be easily 
generated according to the following inflation rule [13]: $\mathrm{A} \rightarrow \mathrm{AB}, \mathrm{B} \rightarrow \mathrm{A}$. The sequence $F_{N}$, with generation number $N$, starts with an arbitrary seed element, $\mathrm{F}_{0}=\mathrm{A}$ for instance, and the inflation rule is repeatedly applied to obtain $\mathrm{F}_{1}=\mathrm{AB}, \mathrm{F}_{2}=\mathrm{ABA}, \mathrm{F}_{3}=\mathrm{ABAAB}$, etc., which displays the well-known Fibonacci symmetry, $\mathrm{F}_{N+1}=\left\{\mathrm{F}_{N}, \mathrm{~F}_{N-1}\right\}$ for $N=1$.

Now we consider a TE-polarized monochromatic wave to be incident normally on the $n$-th generation Fibonacci multilayer $(n \mathrm{GFM})$ in $Z$ direction. We use the transfer-matrix method [14] to calculate the transmitted spectrum and the electric field intensity distribution in the structure.

Figure 1 shows the linear transmission spectra of a light beam into the Fibonacci multilayered photonic structure as a function of the frequency. We have chosen medium $\mathrm{B}$ as titanium dioxide $\left(\mathrm{TiO}_{2}\right)$, whose refractive index is $n_{\mathrm{B}}=2.3$ while medium $\mathrm{A}$ (nonlinear material) is considered to have $n_{\mathrm{A}}=n_{0}+n_{2} I$ where $n_{0}=1.5$ and $n_{2}=2.3 \times 10^{-10} \mathrm{~cm}^{2} / \mathrm{W}$ as in Ref. [15]. The thicknesses $d_{\mathrm{A}}$ and $d_{\mathrm{B}}$ are chosen according to $d_{\mathrm{B}}=\lambda_{0} / 4 n_{\mathrm{B}} \approx 43 \mathrm{~nm}$ and $d_{\mathrm{A}}=\lambda_{0} / 4 n_{\mathrm{A}} \approx 67 \mathrm{~nm}$ where $\lambda_{0} \approx 400 \mathrm{~nm}$. We can indicate several important features shown in Fig. 1.

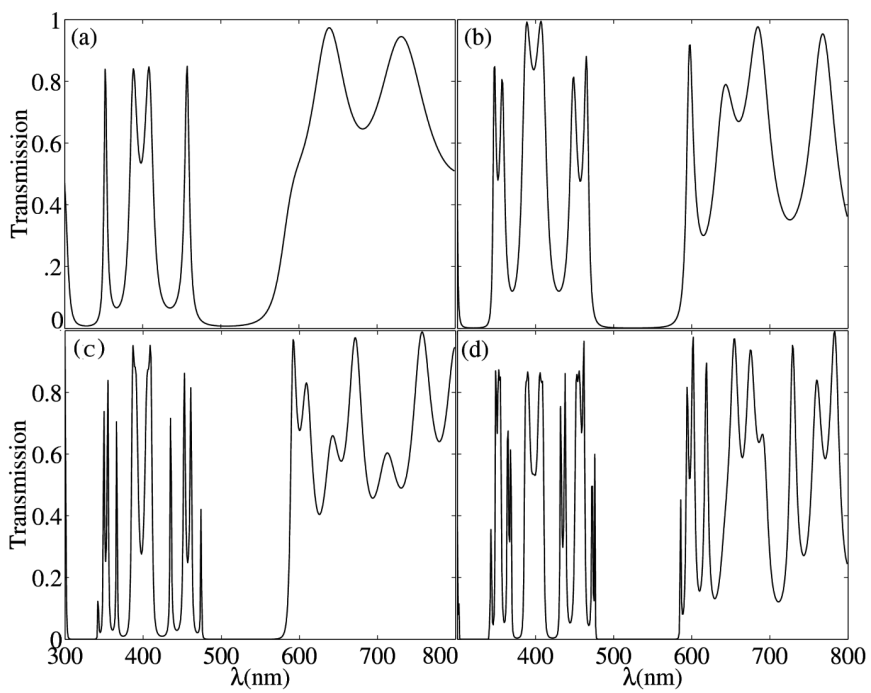

Fig. 1. Linear transmission spectra for right (or left) incidence versus wavelength with $d_{\mathrm{A}}=67 \mathrm{~nm}$ and $d_{\mathrm{B}}=$ $43 \mathrm{~nm}$ for (a) 7GFM, (b) 8GFM, (c) $9 \mathrm{GFM}$, (d) 10GFM.

First, the transmission spectrum of a Fibonacci system contains forbidden frequency regions called "pseudo band gaps" similar to the band gaps of a photonic crystal, these band gaps have gradual formalism for $j \geq 7$.

Second, it is clear that the transmission spectrum has self-similar feature around $\lambda_{0}$. This is one of interesting optical properties of quasicrystals. Also this structure shows high (perfect and near perfect) transmission resonances. Such perfect transmission resonances in asymmetric structures are promising in designing nonreciprocal optical devices such as nonlinear all-optical diodes.

In Fig. 2 we investigate the linear responses of the

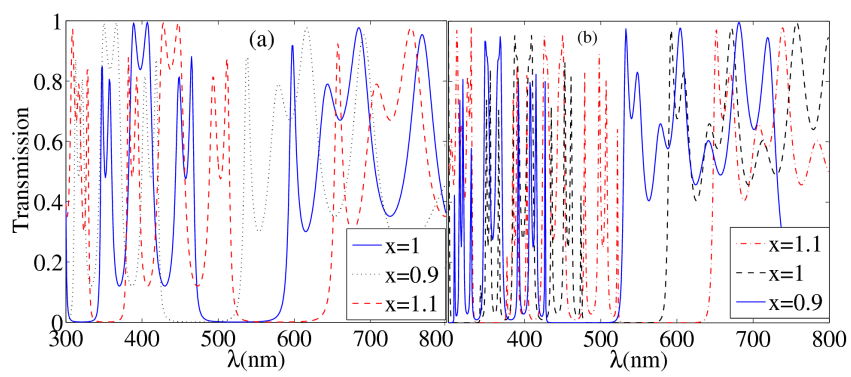

Fig. 2. The dependence of the transmission on the scaling at the (a) 8 GFM and (b) 9 GFM with the same set of parameters as in Fig. 1.

quasiperiodic Fibonacci structure when the system undergoes scaling, i.e. $d_{\mathrm{A}}, d_{\mathrm{B}} \rightarrow x d_{\mathrm{A}}, x d_{\mathrm{B}}$, where $x$ denotes the scaling ratio. We can observe that, when the structure undergoes a small scaling $(x=0.9)$, the transmission profile shifts to left and shifts to right when its undergoes the scaling $x=1.1$, so we can control the location of the perfect resonances by changing the thicknesses of the layers independently, so we can approach perfect resonances in each desired wavelength.

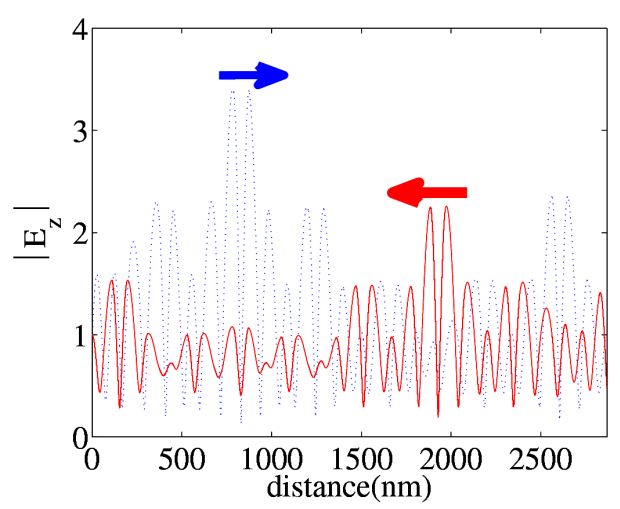

Fig. 3. The electric field intensity distribution in the 9GFM for right (solid line) and left (dashed line) incidences at wavelength $400 \mathrm{~nm}$ with the same set of parameters as in Fig. 1.

Figure 3 shows the electric field distribution in the 9GFM for two opposite incident directions. The electric field intensity distribution for left incident direction is different from the other; also the electric field intensity localized for left incidence is larger than for right incidence so we can say that the nonlinear effect in the structure for left incidence is excited at lower intensities than that for right incidence. It leads to transmission becoming sensitive to the propagation direction.

\section{Bistability and all-optical diode action}

At last we study the nonlinear transmission of $7 \mathrm{GFM}$, $8 \mathrm{GFM}$, 9GFM, and 10GFM as a function of input intensity for forward and backward incidences. As illustrated by Fig. 4 the structures exhibit optical bistable 
behavior for both right and left incidences, but bistability threshold occurs in different intensities for opposite incidence directions. In the region between two bistability thresholds of two incident directions, in the 7GFM e.g. the transmission of left incident light is at the upper branches due to its intensity beyond bistability threshold, whereas the transmission of right incident light is at the lower branches due to its intensity below bistability threshold. This strong nonreciprocal transmission can be useful for AODs. There are two key characteristics for all-optical diodes: low operating power and high transmission contrast, now we calculate the optimal transmission contrast for the considered structures.

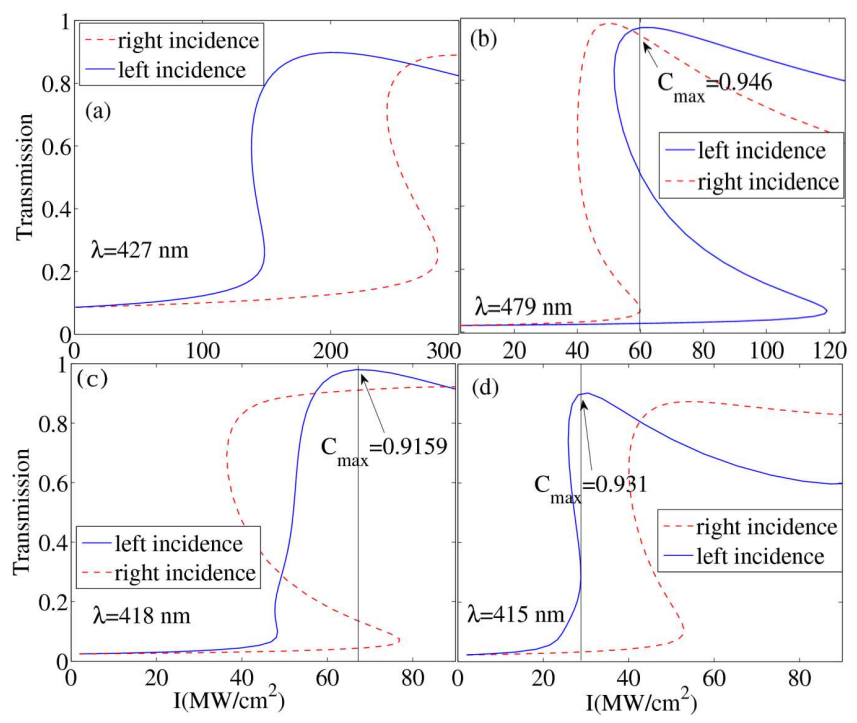

Fig. 4. Nonlinear transmission versus input intensity for right and left incident directions for (a) 7GFM, (b) $8 \mathrm{GFM}$, (c) $9 \mathrm{GFM}$, and (d) 10GFM at the illustrated pump wavelengths.

Transmission contrast has been defined as $C=\left(T_{\text {left }}-\right.$ $\left.T_{\text {right }}\right) /\left(T_{\text {left }}+T_{\text {right }}\right)$ to characterize efficiency of AODs by Gallo et al. [16]. From Fig. 4 it is seen that we can achieve low operating input intensities by increasing the generation number of structure. Also the optimal transmission contrast increases and occurs in higher intensities by increasing the wavelength. So it is possible to achieve higher contrast transmission by increasing the pump wavelength. However, it leads to increase in operating input intensity. For instance, in Fig. 4c the optimal transmission contrast is 0.916 and it occurs at input intensity $67.5 \mathrm{MW} / \mathrm{cm}^{2}$ at the wavelength $418 \mathrm{~nm}$. By increasing the wavelength to $420 \mathrm{~nm}$ and with input intensity $83.5 \mathrm{MW} / \mathrm{cm}^{2}$, it will be 0.957 . Achieving low operating input intensity is highly desirable to the practical application of AODs. In this paper our analysis has shown the feasibility of the Fibonacci quasiperiodic nanostructures as AODs. These structures show high unidirectional transmission with low operating intensities, which is the merit of the structures for design of AODs. Moreover, 7GFM, 8GFM, 9GFM, and 10GFM are composed of $21,34,55$, and 89 layers, respectively. So the results show that these structures are compact and it is the other merit of these structures.

\section{Conclusions}

In this paper we have theoretically studied the optical properties of the Fibonacci quasiperiodic nanostructures. By studying the variation of the nonlinear transmission with light input intensity, for two opposite incident directions, we have found that these structures exhibit high unidirectional transmission due to high linear transmission as shown in Fig. 1 and high transmission contrast due to the strong asymmetry of the Fibonacci sequence. Asymmetry of structure increases by increasing the generation number. So it is seen that the optimal transmission contrast increases by increasing the generation number. Also it occurs in low intensities. So these structures as the all-optical diode have high efficiency in the nonreciprocation. In this connection, we expect that our studies would be helpful to create compact nonreciprocal devices and all-optical diodes in visible regime.

\section{References}

[1] F. Biancalana, Appl. Phys. 104, 093113 (2008).

[2] C. Xue, H. Jiang, H. Chen, Opt. Expr. 7, 7479 (2010).

[3] S.F. Mingaleev, Y.S. Kivshar, J. Opt. Soc. Am. B 19, 2241 (2002).

[4] M.W. Feise, I.V. Shadrivov, Y.S. Kivshar, Phys. Rev. E Stat. Nonlin. Soft Matter Phys. 71, 037602 (2005)

[5] R. Philip, M. Anija, Ch.S. Yelleswarapu, D.V.G.L.N. Rao, Appl. Phys. Lett. 91, 141118 (2007).

[6] X. Hu, Z. Li, J. Zhang, H. Yang, O. Gong, Adv. Funct. Mater. 21, 1803 (2011).

[7] N. Kono, K. Kakihara, K. Saitoh, M. Koshiba, Opt. Expr. 15, 7737 (2007).

[8] M.E. Zoorob, M.D.B. Charlton, G.J. Parker J.J. Baumberg, M.C. Netti, Nature 404, 740 (2000).

[9] E.L. Albuquerque, M.G. Cottam, Polaritons in Periodic and Quasiperiodic Structures, Elsevier, Amsterdam 2004.

[10] J.M. Luck, C. Godreche, A. Janner, T. Janssen, J. Phys. A: Math. General 26, 1951 (1993)

[11] M. Werchner, M. Kira, S.W. Koch, J. Sweet, J.D. Olitzky, J. Hendrickson, B.C. Richards, G. Khitrova, H.M. Gibbs, A.N. Poddubny, E.L. Ivchenko, M. Voronov, M. Wegener, Opt. Expr. 17, 6813 (2009).

[12] M. Kolar, Phys. Rev. B 47, 5489 (1993)

[13] M. Kohmoto, B. Sutherland, C. Tang, Phys. Rev. B 35, 1020 (1987)

[14] S.M. Wang, L. Gao, Opt. Commun. 267, 197 (2006).

[15] R.W. Boyd, Nonlinear Optics, 3th ed., Academic Press, Orlando 2007.

[16] K. Gallo, G. Assanto, K.R. Parameswaran, M.M. Fejer, Appl. Phys. Lett. 79, 314 (2001) 